Woch., 1893, No. 30) has found benzonapthol of the greatest service in various dyspeptic disturbances, flatulence and chronic meteorism from intestinal atony, particularly when associated with bismuth subnitrate and resorcin. He gives 8 grains every two hours. However, the remedy is not absolutely infallible; furthermore, in a few exceptional cases of Ewald's, intestinal colic and diarrhea supervened, but promptly disappeared on discontinuing the medica. tion.

M. Bernitt (Wiener Med. Presse, 1892, No. 51), Bruck (Pester Med. Presse, 1892, No. 46), and Zinnis (Deutsche Med. Woch., 1892, No. 30) recommend benzonaphthol in infectious gastro-intestinal diseases of children and babes, while Kuhn of Giessen (Deutsche Med. Woch., 1893, No. 19) denies it all virtues in these affections.

The writer has found benzonaphthol an efficacious antizymotic in conditions characterized by inordinate fermentation, whether functional or inflammatory in nature. The dose for adults is from 8 to 10 grains every two hours, or about 80 grains a day, administered best in cachets; for children up to one year of age, from $1 / 2$ to 2 grains every few hours; from one to three years, five times daily, 3 grains; from four to seven years, 24 grains per day; from eight to fourteen years, 30 grains daily, with an equal part of sugar or a trace of saccharin. In chronic cases full action sets in only after four or five days; its astringent properties are not very pronounced.

I believe I have alluded to all the antidiarrheal remedies introduced during the last few years. Should any member desire information regarding any other intestinal remedies, the writer of this paper shall deem it a pleasure to supply the desired data so far as lies in his power.

13 University Place.

\section{'THE MEDICINAL TREATMENT OF SOME DIGESTIVE DISEASES.}

Presented to the Section on Materia Medica, Pharmacy and Therapeutic at the Forty-ninth Annual meeting of the American Medical Association, held at Denver, Colo., June 7-10, 1898.

BY A. L. BENEDICT, A.M., M.D.,

PROFESSOR OF PHYSIOLOGY AND DIGESTIVE DISEASES, DENTAL DEPARTMENT UNIVERSITY OF BUFFALO, BUFFALO, N. Y.

There has always been a tendency, both within and without the medical profession, to scoff at drugs. The tremendous development of certain scientific and mechanical departments of the healing art, within the last two decades has, by contrast, placed the homely skill of using medicines in the background. Teachers and students of medicine have alike neglected the old-fashioned details of therapeutios; therapeutic nihilists have arisen who have sought to discredit the value of drugs and, naturally enough, the preconceived notion that ponderable therapeutic agents have little use, has made it possible for those who hold such a view to demonstrate their belief from their own experience. The writer is sufficient of an old fogy to hold that if the regular profession would devote to the physiologic and therapeutic study of drugs the same amount of pains that the homeopaths bestow upon what we believe to be false theories, a great advance would follow, and internal medicine would be correspondingly dignified.

Diserse is, in the main, a chemic disturbance, though often of so recondite a nature that we are forced to employ some such non-committal term as "vital" and to abandon all thought of cure through the agency of an ordinary chemic reaction. Even in this latter kind of disturbances, we often find a wellestablished antagonism between diserse and drug, which can not be explained but to which we allude under the term "selective affinity." In general, it may be said that a priori, there is just as much rationality in expecting relief from the action of a chemical circulating in the blood as from the effervescence of a briny bath, or the hanging of a nervous case by the chin and occiput till cured, or the kneading of dermal and subcutaneous tissues, or from reducing a human being to a vegetative state, all of which are methods of therapeutics in good repute and of great value.

Digestive disturbances afford an especially favorable field for the application of drugs, 1, because they are usually more or less chronic and yet not suffi. ciently urgent to induce the patient to make the sacrifice of time and business interests demanded by hygienic therapeutics; 2 , because an exact diagnosis, at least of the immediate physiologic failure, is usually possible, though requiring skill and special meth. ods of examination; 3 , because these bnormalities are, to a far greater degree than those of other organs, either chemic or vital in so simple a way as to be amenable to drugs; 4 , because surgical treatment is applicable to but few cases and, to these, with a grave prognosis; 5 , because really valuable means of local therapeutics have been greatly abused, as to technique and frequency of employment.

For practical purposes the majority of gastric disturbances may be considered functional. The writer's personal opinion is that they are much more frequently dependent on organic change than is usually supposed, but as death seldom occurs, and as postmortem changes can very rarely be prevented or anticipated, for obvious sentimental if not better reasons, we know very little about the actual state of the gastric mucosa in dyspepsia. Even the microscopic examination of particles of epithelium obtained by siphonage or spontaneous vomiting, is not reliable, since there is normally a shedding of more or less degenerated epithelium. Probably 70 per cent. of gastric diseases, taking them as they occur, are essentially atonic dyspepsias, characterized by deficiency of hydrochloric acid and by sluggish motor action. The immediate relief of the former condition is ideally fulfilled by the administration of the same acid, while without obvious catarrhal involvement, local treatment is not indicated. As to the motor function, probably nine out of ten physicians who were thoroughly impartial, that is to say inexperienced, would declare that the ideal treatment was the intraventral use of faradism. But, considering carefully the various experimental studies as to the action of electricity on the muscle of the stomach, we must conclude that, while it is not absolutely impossible to note a contraction during the time that the battery is in use, there is no evidence that the faradism stimulates the gastric muscle to contract when subjected to this method of treatment. On the other hand, we have a drug, strychnin, which most certainly does stimulate smooth muscle, whether of the stomach or elsewhere in the body, and whether we depend upon physiologic experiments or clinical experience. Such cases-and others--might be thought suitable for the employment of hygiene. But we find that ordinary gymnastic exercises do not produce much improve- 
ment; that the fresh air of the country or sea-side is bowel as well as the stomach, salacetol is of the highoverbalanced by the difficulty of finding proper food est rank. The writer's experience is not so favorable in either the typic farmhouse or hotel. Heaven for- for the benzo-compounds though these are also antibid that we should expose such patients to the bacte- septic. Hydrogen peroxid is especially valuable when rial and mental infection of a sanitarium or that we there is muco-purulent matter present.

should initiate them into the mysteries of invalidism by prescribing massage and rest-cure. They may be cured with great éclat by lavage, but they simply do not need to subject themselves to the discomfort and expense of this method, unless the case is extreme. Neither are they fit subjects for the believer in the elaborate simplicity of dietetics. Some few atrocities of modern cookery may be forbidden but, as a rule, the stomach needs the stimulation of varied and appetizing food, and the more such patients are dieted the more they have to be, the gastric power failing concomitantly with the diminution of the task set before it. Not rarely we find patients who are relieved by hydrochloric acid and strychnin but who do not regain the power to digest without this assistance. Then hygiene, change of air and scene, rest and recreation, come into play, but they must follow, not precede, medication.

In the rather rare cases in which the stomach produces too much acid, avoidance of chlorids, of stimu. lating food and of irritating circumstances of all kinds, are the prime indications. If treatment, except in a negative sense, is needed, alkalies and secretory depressants are efficient. Rest is undoubtedly the ultimate need of most such patients, though there are a few who are lithemic, and who need some offset to idleness and high living. Very rarely indeed is the rest-cure indicated, there being a great difference between rest and rest-cure.

As to ferments, the following general statements may be made: 1 , there is no information or reasonable supposition that a ferment of the digestive glands is ever produced in excess; 2, the failure of a ferment occurs only in the most serious organic disturbances - though not necessarily locally organic, thus, fever is often attended with failure of ferments. The salivary ferment is particularly resistant to disease, though not of very great importance in health; 3 , the administration of ferments should never be resorted to without first demonstrating their absence; 4, after proving that a ferment really is deficient-something that occurs in scarcely 1 per cent. of cases-we must ask whether the stomach and intestine are in a condition to digest on the mere supplying of the ferment, or whether predigestion, rectal nutrition, etc., are not indicated. As a rule, the body which lacks ferments lacks the power of using them if artificially supplied.

Gastric catarrb, ulcer and cancer, for many therapeutic considerations, duplioate the indications of purely functional dyspepsia, if such a thing exists. The lack or excess of acid, the need of a motor tonic, the indication for an antiseptic or anesthetic, must be worked out, case by case, without attempting to rely on a general rule. While a stomach which is badly dilated and diseased so that food actually ferments and putrefies can be cleansed only by repeated lavage, there are efficient internal antiseptics which are all that are needed for the milder cases, and which usu-. ally are indicated in addition to lavage, in the severer conditions. All things considered, oil of peppermint is the best of these, but oil of wintergreen may be used if there is a joint indication-no pun is intended -for the employment of a salicylate. For more gradual antiseptic action, when we must consider the

Surgery suggests itself mainly in the relief of gastric cancer and ulcer. With the former, the theoretic indication for surgery is balanced by the difficulty of making a diagnosis in time and by the grave prognosis, so far as the operation itself is concermed, so that at present the average case of cancer will continue to receive mainly medical attention. Although the result is inevitably fatal, it must not be forgotten that the duty of the physician is all the more urgent to maintain comfort, and that the ordinary gastric cancer does not occasion the sharp pain that is too frequently connected with malignant disease; hence there is much room for rational treatment in improving digestive deficiencies, in maintaining gastric motility, in keeping the chyme aseptic and even in obtaining healing of malignant ulcerations. Morphin is by no means a drug to be freely used. Gastric ulcer, superficially considered, might be thought to indicate ligation of the bleeding part, but medical and surgical opinion is very nearly unanimous that operation is not feasible. The local use of styptics is plainly contraindicated; first, because it is impossible to get them to the site of hemorrhage in sufficient quantity and sufficient concentration; secondly, because they are all irritants, and tend to cause retching, which aggravates the primary trouble. The real indications are two-absolute quiet obtained by morphin, and vascular constriction by ergot. Both should be given hypodermically. To the best of the writer's knowledge, there is no satisfactory preparation of ergot for such use, all being irritating and some of the so-called ergotins being of unfixed dosage. On the whole, the official fluid extract is about as good as the more expensive preparations. Few physicians realize that many of the unpleasant symptoms and part of the actual danger of gastric ulcer are due to absorption of decomposed blood. Indican is found in the urine in enormous amount and there is great need of intestinal asepsis. Still, for the first twenty-four hours at least, the need of quiet is so urgent as to forbid cathartics, enemata, or even intestinal antiseptics. A little ice is usually given, simply to keep the patient from being too restless, but even this is to be avoided as far as possible. After a day or two, salacetol or bismuth subgallate may be given and, after the bowels have been freed from blood, it is well to give bismuth systematically, rather as an astringent dressing than as an antiseptic. The writer's preference is for a 10 per cent. emulsion of bismuth hydrate in water, or pure liquid petrolatum, if the patient shows no distaste for the mineral oil.

In gastric catarrh, with or without dilatation, bismuth subgallate is an excellent and efficient remedy, though by no means able to supplant lavage and inflation with menthol or similar sprays. In any condition in which the gastric mucosa is involved, we must make a sharp distinction between the needs of the stomach itself and the needs of the digestive process. Usually, there exists quite a definite opposition between these two indications, the more or less alkaline and astringent application which is generally needed for the gastric wall interfering with digestion, while the hydrochloric acid and secretory and motor stimulants required to assist digestion are somewhat 
irritant to the diseased mucosa. In cases that are absolutely or practically functional, we naturally pay attention only to the digestive deficiencies; in severe organic diseases, such as acute catarrh, ulcer and some manifestations of cancer and chronic catarrh, gastric digestion must be discontinued entirely for at least a few days, the body being nourished by the bowel and skin while the stomach is being medicated. A large number of cases fall between these two extremes and, as a number of favorite ready-made tablets and liquid mixtures show, many physicians simply stir together the various drugs indicated and administer them all at once, letting their diverse activities battle for the supremacy. In such cases, the rational treatment consists in doing one thing at a time. Give such foods as may be digested in, say, three hours; in other words, rather plain but still appetizing and ordinary articles of food. Administer such digestive aids as shall insure digestion and emptying of the stomach in about this time. Then, every morning and afternoon, a period of two or three hours is at our disposal for medicating the gastric wall, while a drug administered at bed-time has a much longer period of possible action. The writer would confess that his conception of a drug acting on the stomach and bowel itself is pretty nearly limited to bismuth. The various vegetable astringents lack the property of a powder dressing; silver, copper and zinc are too active for more than temporary use, and each, moreover, possesses special objectionable features. Cerium oxalate has no obvious advantage over bismuth, while, in most of the cases in which one or the other must be used, there is altogether too much oxalic acid formation in the system. The main point in administering bismuth is to give enough of it. With the possible exception of some of the newer antiseptic compounds, the minimum dose should be half a gram, and twice or three times this amount should usually be given. Yet, within a few days, a cautious druggist has telephoned the writer in regard to overdose in the case of a prescription calling for a gram of the subgallate, three times a day.

For the long reach of bowel between the accessible portions of the alimentary canal, the same general principles of medication apply as to the stomach. It is of the greatest importance to be sure that the intestinal disturbance is not the final breaking down of the power to perform vicariously the duties of the stomach. Many stomachs habitually shirk digestion, and the owner is unaware of the suspension of function so long as the gastric motor power is sufficient to pass the chyme on to the duodenum. Next, by high enemata, we must keep the lower bowel clean and healthy. Finally, we must administer the slowly. soluble antiseptics, occasionally ferments inclosed in stomachproof coatings, and usually the astringent bismuth, preferably now in pure petrolatum. Juggling with cathartics and constipating drugs is not therapeutics, although it may be an inevitable makeshift.

Although in scolecitis, surgery affords the safest and most thorough treatment, there are certainly many mild cases which recover under either constipating medication with opium, or laxative treatment with salines or belladonna, with or without intestinal antisepsis and hydrotherapy. If we could be sure that free drainage existed from the mouth of the appendix and that gangrene and virulent ulceration could be excluded, medical treatment of even acute cases might be justifiable. But, at present, the only cases in which operation should not be insisted upon, are mild and more or less chronic ones, the patient being so situated that surgical aid may be called almost at a moment's notice. If we carefully consider the history of scolecitis cases, we must conclude that not grape seeds, nor other foreign bodies, not consti. pation, not necessarily catarrh of the bowel, not taking cold, not even anatomic peculiarities as to meso-, blood-vessels, and lymphoid tissue, are the essential causes. The real cause, in the writer's belief, is unusual virulence of intestinal bacteria, marked by prolonged intestinal indigestion and fermentation. Thus, there is a rational field for the use of antiseptics, drugs to regulate the proper discharge from the bowels, including water, and, in addition, some of the slowly soluble compounds of iodin as resorptives.

In our appreciation of the seriousness of scolecitis and the danger of too long delay of operation, we have gone rather too far in abandoning the old-fash. ioned conception of typhlitis. There most certainly do exist cases of catarrh and atony, especially of the head of the colon, though not absolutely limited by an arbitrary anatomic division. Here we find the sausage-shaped fecal tumor just as truly as did our predecessors, and, in the absence of leucocystosis, of a septic temperature and pulse, we have as plainly as ever an indication for medical treatment, including salines, antiseptics and, later, such means as will check the tendency to catarrh and prevent an atonic yielding of the bowel.

\section{THE TREATMENT OF HEART DISEASE BY SALINE BATHS AND RESISTED MOVE- MENTS-SCHOTT METHOD.}

Presented to the Section on Materia Medica, Pharmacy and Therapeutics at the Forty-ninth Annual Meeting of the American Medical Association, held at Denver, Colo., June 7-10, 1898. BY CHARLES LYMAN GREENE, M.D.

CLINICAL PROFESSOR OF MEDICINE AND PHYSICAL DIAGNOSIS, MEDICAL DEPARTMENT OF THE UNIVERSTTY OF MINNESOTA, VISITING PHYSICTAN TO THE CITY AND COUNTY HOSPITAL AND ST. PAUL FREE DISPENSARY.

$$
\text { ST. PAUL, MINx. }
$$

The value of a therapeutic agent or method can not be measured by the volume of its literature nor yet by the enthusiasm of its advocates, and no doubt too great a measure of faith in the testimony of false witnesses has bred in us that skepticism which, however admirable as a mental attitude in scientitic work, may nevertheless, if too greatly expanded, become a serious stumbling-block in the path of medical progress. We are right in insisting upon something more than dog. matic assertion or the voice of authority in the determination of therapeutic values; we may justly demand the right to withhold judgment, ask for substantial proofs and thoroughly sift our evidence, but behind all this there must be a sincere and earnest desire, not alone to detect error, but also to know the truth, and the ultimate test must after all be derived from personal experience and the repetition of such xperiences.

If a new procedure is rational, if the evidence in its favor seems fairly good, and if it promises more than the old, we are in duty bound to give our patients the benefit of the trial. The world has not moved very greatly in this direction in the last 200 years. We may now read with astonishment the vituperative paragraphs which proved with a most imposing show of logic and a vast amount of physiologic and anatomic 